\title{
EFEITOS DO DIMETILSULFÓXIDO NO ESTRESSE OXIDATIVO E NA REGENERAÇÃO HEPÁTICA PÓS-HEPATECTOMIA EM RATOS
}

\author{
EFFECTS OF DIMETHYLSULFOXIDE ON POST-HEPATECTOMY OXIDATIVE STRESS \\ AND LIVER REGENERATION IN RATS
}

\author{
José Ulisses de Souza Melo, TCBC-CE'; Paulo Roberto Leitão de Vasconcelos ${ }^{2}$; \\ Jefferson Menezes Viana Santos³; Manoel Messias Campos Júnior³; \\ Marcus Vinicius Amaral Barreto ${ }^{3}$; Osamu de Sandes Kimura ${ }^{3}$
}

\begin{abstract}
RESUMO: Objetivo: avaliar a influência do DMSO sobre o estresse oxidativo e a regeneração hepática pós-HP via um modelo experimental. Método: 36 ratos Wistar machos jovens foram aleatoriamente distribuídos em dois Grupos de 18 animais: parcialmente hepatectomizados com infusão diária de solução salina (controle) e parcialmente hepatectomizados com aporte diário intraperitoneal de DMSO, todos por duas semanas. Nos tempos 36h (T1), 168h (T2) e 336h (T3) pós-HP, glutationa (GSH) foi medida no plasma e no tecido hepático, enquanto glicose e bilirrubina total foram aquilatados no sangue. A massa do fígado residual, nos mesmos tempos, foi o parâmetro utilizado para estimar a evolução da regeneração do fígado. Resultados: DMSO baixou os níveis de GSH hepático e sangüíneo mas não interferiu na evolução da massa em regeneração. Conclusão: DMSO inibiu o estresse oxidativo pós-HP mas não mostrou alterações significantes na regeneração hepática em ratos (Rev. Col. Bras. Cir. 2008; 35(2): 103-108).
\end{abstract}

Descritores: Hepatectomia; Estresse oxidativo; Ratos; Regeneração hepática; Peroxidação de lipídeos; Dimetil sulfóxido.

\section{INTRODUÇÃO}

A notável capacidade de regeneração do fígado dos mamíferos já é conhecida há muito tempo e, no rato, após ressecção dos lobos lateral esquerdo e mediano (HP), os quais representam aproximadamente $67 \%$ da massa hepática total ${ }^{1}$, os lobos residuais, lateral direito e caudato, deflagram uma resposta essencialmente hiperplásica com regeneração de células e tecidos que culminam, em 3 a 14 dias, na restauração do volume original da glândula ${ }^{2,3,4}$. Muitas substâncias têm sido experimentadas em animais parcialmente hepatectomizados para avaliar as suas atuações no processo regenerativo $^{5,6}$ e no estresse oxidativo induzido pela $\mathrm{HP}$, vez que a peroxidação lipídica é um dos fatores na enorme gama de influenciadores do fenômeno da regeneração ${ }^{7,8,9}$.

O dimetilsulfóxido (DMSO) é um líquido orgânico, límpido, incolor, ligeiramente oleoso, sem odor em sua forma pura, descoberto em 1867 quando da preparação da polpa de madeira utilizada pelos fabricantes de papel ${ }^{10}$. É um composto derivado da lignina, substância responsável pela rigidez das células vegetais. Sem utilização específica até a Segunda Grande Guerra, seu uso como solvente industrial e comercial se intensificou marcadamente no pós-guerra e, em 1961 foi utilizado como fármaco, pela primeira vez, por Stanley W. Jacob, coordenador do programa de transplantes de órgãos da
Oregon Health Sciences University (Portland, USA) ao pesquisar sua potencialidade na preservação de órgãos ${ }^{11}$. Sua molécula, $\mathrm{C}_{2} \mathrm{H}_{6} \mathrm{SO}$, é anfipática com grande domínio polar e a presença de dois grupos metil, apolares, resulta em solubilidade tanto em meio aquoso como em meio orgânico ${ }^{12}$, possuindo a capacidade de penetrar, com extrema facilidade, em órgãos, tecidos e membranas, inclusive celulares e intracelulares ${ }^{13,14,15}$, o que se deve essencialmente a esta sua natureza polar, ao pequeno tamanho de sua molécula e à sua compactada estrutura polimolecular ${ }^{15}$. Trabalhos atestam que DMSO rapidamente se distribui, após injeção intraperitoneal (i.p.), em todos os órgãos e tecidos ${ }^{14}$ e possui intensa ação antioxidante via seqüestro de radicais livres ${ }^{16,17,18}$. No entanto, DMSO não é isento de efeitos colaterais: mesmo administrado em pequenas doses, pode produzir efeitos tóxicos ao fígado e, em doses maiores pode provocar esteatose hepática ${ }^{19}$.

O presente trabalho objetiva avaliar a influência do DMSO sobre o estresse oxidativo e a regeneração hepática pós-HP via um modelo experimental devido a Higgins \& Anderson ${ }^{2}$.

\section{MÉTODO}

A presente pesquisa obedeceu às normas do Colégio Brasileiro de Experimentação Animal (COBEA) e foi apro-

\footnotetext{
1. Doutor em Cirurgia pela Universidade Federal do Ceará - UFC; Master of Science, NYU-USA; Mestre em Matemática, pela UnB; Professor Adjunto IV do Departamento de Cirurgia da Universidade Federal do Ceará (UFC).

2. Ph.D., Inglaterra; Professor Associado de Cirurgia, UFC; Coordenador do Programa de Pós-Graduação Stricto sensu em Cirurgia da UFC.

3. Acadêmico de Medicina da Universidade Federal do Ceará - UFC.

Recebido em 15/10/2007

Aceito para publicação em 14/10/2007

Conflito de interesses: nenhum

Fonte de financiamento: nenhuma

Trabalho realizado no Laboratório de Cirurgia Experimental (LABCEX) do Programa de Pós-Graduação Stricto sensu em Cirurgia da UFC.
} 
vada pela Comissão de Ética em Pesquisa Animal (CEPA) da UFC sob o número protocolar 14/06 de 11 de Agosto de 2006.

Foram utilizados 36 ratos machos, jovens ( $70 \pm 10$ dias), (Rattus norvegicus: var. albinus, Rodentia, mammalia) da linhagem Wistar, com peso médio de $135,54 \mathrm{~g}$ provenientes do Biotério Central da Universiade Federal do Ceará (UFC) e albergados, para o estudo, no biotério do LABCEX do Departamento de Cirurgia da UFC em gaiolas de prolipropileno com tampa de aço inoxidável e piso forrado com maravalhas, deixando aproximadamente $250 \mathrm{~cm}^{2}$ de área livre. Os animais foram mantidos em ciclos circadianos de claro/escuro, com 12 horas de luz de baixa intensidade e 12 horas de escuridão, tanto antes como após os procedimentos cirúrgicos e por todo o período da experimentação. As gaiolas foram higienizadas e os animais examinados cotidianamente. A temperatura ambiente foi mantida entre 18 e $27^{\circ} \mathrm{C}$, a umidade relativa do ar entre 40 e $70 \%$ e foi livre o acesso à água e à ração balanceada própria para a espécie composta de $4 \%$ de lipídios, $21 \%$ de proteínas, $52 \%$ de carboidratos e o restante de resíduos não digeríveis (Guabi Nutrilabor ${ }^{\circledR}$, Mogiana Alimentos - São Paulo, S.P.)

Os animais foram distribuídos ao acaso em dois grupos de 18 ratos. No Grupo G1 - Grupo controle - os animais foram submetidos à HP no tempo T0 e, de imediato, submetidos à infusão diária de solução salina a $0,9 \%$ (SF, salina). Em cada um dos tempos T1(36h), T2(168h) e T3(336h) pós-HP, um lote de seis ratos deste Grupo, tomados ao acaso, foram anestesiados e submetidos à relaparotomia, à coleta de sangue por punção da veia cava abdominal sob visão direta e, em seguida, à hepatectomia complementar (HC) e então sacrificados via dose letal inalatória de éter dietílico. $\mathrm{O}$ fígado foi então pesado e, para avaliação da glutationa (GSH), congelado a $78^{\circ} \mathrm{C}$. O sangue foi coletado em dois tubos de ensaio: um para as aferições da glicose e da bilirrubina e outro para as mensurações da GSH plasmática e tecidual. No Grupo G2, Grupo-teste, os ratos foram submetidos exatamente aos mesmos procedimentos do grupo controle com uma única diferença: em vez de salina, a administração intraperitoneal diária foi de DMSO.No tempo T0, logo antes da intervenção cirúrgica, os animais foram pesados individualmente, dado importante para o cálculo das dosagens de SF e DMSO infundidas. Os animais não foram submetidos a qualquer tipo de restrição (água, alimento etc.) antes do procedimento cirúrgico. O SF 0,9\% foi fabricado pela Química Farmacêutica Gaspar Viana (Fortaleza, CE) contendo em cada $100 \mathrm{~mL}$ : cloreto de sódio 0,9g + água purificada q.s.p. 100mL. O DMSO utilizado foi adquirido da Cromato Produtos Químicos Ltda (Diadema, S.P) com as caracerísticas: Aparência/Estado físico: líquido claro e incolor. Pureza: $99 \%$. Peso molecular: 78,13. Densidade: 1,1004 $\mathrm{g} / \mathrm{cm}^{3}$. Cada $\mathrm{mL}$ do líquido contém $1,10 \mathrm{~g}$ de DMSO. Ponto de Fusão: $18,5^{\circ} \mathrm{C}\left(292^{\circ} \mathrm{K}\right)$. Ponto de ebulição: $189^{\circ} \mathrm{C}\left(462^{\circ} \mathrm{K}\right)$. Viscosidade: $1,996 \mathrm{cP} \mathrm{a} 20^{\circ} \mathrm{C}$. Momento Dipolar: 3,96 D. Solubilidade: em água, etanol, benzeno e clorofórmio. O DMSO foi administrado como solução aquosa a $3 \%$ obtida volume por volume pela junção de $97 \mathrm{~mL}$ de SF a $0,9 \%$ com $3 \mathrm{~mL}$ de DMSO puro. Cada $\mathrm{mL}$ da solução resultante continha 3,3mg de DMSO e a dose diária (d.d.) utilizada foi de $0,1 \mathrm{~mL}$ para cada $100 \mathrm{mg}$ do rato, d.d. esta igualmente fornecida de salina em G1. Imediata- mente antes das infusões, a solução de DMSO foi agitada durante meio minuto, com a finalidade de manter a homogeneidade do líquido infundido.

Sob anestesia inalatória de éter dietílico, epilados e degermados com polivinilpirrolidona-iodo, a laparotomia foi realizada via incisão transversa oblíqua subcostal bilateral com concavidade ligeiramente caudal (incisão de Kocher bilateral) com aproximadamente $4 \mathrm{~cm}$ de extensão. Após a abertura da parede abdominal, as alças intestinais foram afastadas e após a colheita de sangue da veia cava por punção em sentido céfalo-caudal à $45^{\circ}$ com agulha hipodérmica $25 \times 7(0,7 \mathrm{~mm} \mathrm{x}$ 25mm, 22Gx1", NIPRO®, Sorocaba, SP) acoplada em seringa de $5 \mathrm{~mL}$ (BD Plastipak ${ }^{\circ}$, Curitiba, PR), identificou-se o fígado e seus lobos, ressecção do ligamento teres hepatis e, em seguida, ligadura com fio de algodão zero do pedículo hepático composto pela veia porta, artéria hepática e via biliar, acima dos lobos posteriores (caudato e lateral direito). Em seqüência, foi feita a secção da tríade portal logo acima da ligadura, com conseqüente ressecção, em bloco, dos lobos anteriores, resultando em uma hepatectomia parcial bilobular de aproximadamente $70 \%$ conforme descrito por Higgins \& Anderson em $1931^{2}$. Realizadas as revisões da cavidade abdominal e da hemostasia, procedeu-se à síntese via laparorrafia em dois planos: o primeiro peritônio-músculo-aponeurótico com chuleio contínuo simples de categute 4.0 e o segundo, o da pele, com pontos separados usando fio de algodão 2.0. Logo em seguida, ainda com o animal anestesiado, foi injetada a primeira dose intraperitoneal (SF em G1e DMSO em G2) no quadrante inferior esquerdo do abdome e, então, o animal andou após a suspensão do anestésico inalatório, permanecendo isolado em gaiola, com a oferta livre de água.

A glicemia e a bilirrubinemia serviram como parâmetros da avaliação do metabolismo hepático enquanto sob regeneração hepática e as medidas do antioxidante GSH, tanto hepático como sangüíneo, para mensurar o estresse oxidativo.

Para a determinação da GSH, calculou-se o teor dos grupos sulfidrílicos não protéicos pela técnica de Sedlak \& Lindsay $^{20}$, o teor de bilrrubina total foi dosado via Meites ${ }^{21}$ e a D-glicose foi determinada segundo o método de Slein ${ }^{22}$.

A hiperplasia celular compensatória dos lobos residuais - a regeneração hepática - após hepatectomia a $70 \%$ foi avaliada pela medidas das massas dos fígados residuais dos ratos nos tempos T1 (36h), T2 (168h) e T3 (336h) posteriores à amputação cirúrgica parcial do fígado. Tais mensurações geraram uma tabela e desta, diagramas de dispersão que, pelo método dos mínimos quadrados, forneceram as retas de regressão (interpolatrizes) apresentadas na Figura1. Com a significância estatística fixada em $95 \%(\mathrm{p}<0,05)$, as interpolatrizes foram comparadas pelo test $\mathrm{t}$ de Student para coeficientes angulares. Tanto o GSH tecidual como o sorológico, bem como a glicemia (Gli) e a bilirrubinemia total (BT), estão apresentados em três gráficos tipo coluna vertical, com a altura refletindo o erro padrão da média (E.P.M.), cada gráfico referente a um dos tempos T1, T2 e T3. Tais gráficos foram obtidos utilizando o soft GraphPad Prism ${ }^{\circledR}$ versão 4.0 de Fevereiro de 2005 (GraphPad Software - San Diego, U.S.A.) com as comparações pelo Teste de Dunnett. 
Evolução Ponderal do Fígado pós-HP

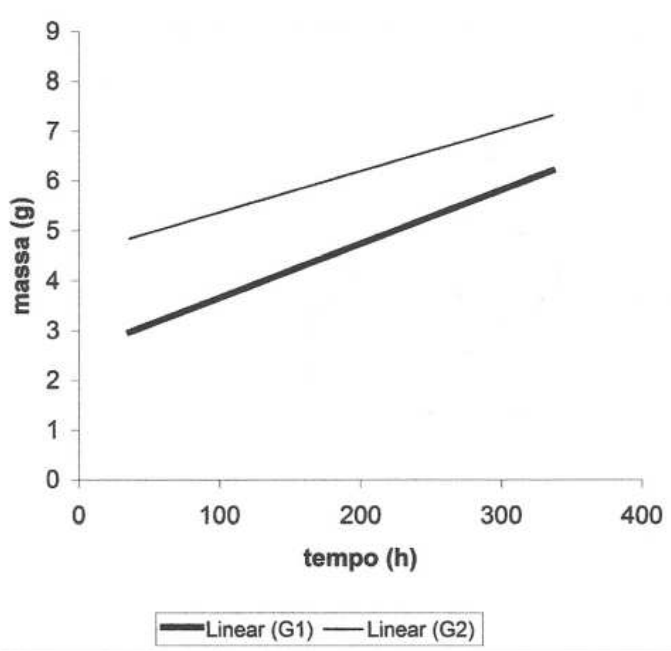

Figura 1 - A regeneração hepática pós-HP não apresentou diferença significante entre os grupos controle G1 experimento G2. (Teste t de Student para coeficientes angulares).

\section{RESULTADOS}

A Figura 1 exibe a evolução do crescimento dos lobos residuais pós-HP e não há diferença estatisticamente significante entre G1 e G2. DMSO, portanto, não interferiu de modo significativo na regeneração hepática pós-HP.

No que se refere à GSH, Gli e BT, somente os gráficos dos tempos que apresentaram diferença significante estão apresentados. GSH hepático diminuiu de forma significante $(\mathrm{p}<0,01)$ nos tempos T2 e T3, conforme pode ser visto respectivamente nas figuras 2 e 3 , o mesmo ocorrendo com os resultados do GSH sangüíneo: figuras 4 e 5 ( $\mathrm{p}<0,01$ e p $<0,005$, respectivamente).

A BT não apresentou quaisquer diferenças significantes em qualquer dos tempos. A glicemia póshepatectomia mostrou-se significativamente menor $(\mathrm{p}<0,01)$ nos tempos T2 e T3 (a figura 6 exibe o tempo T2).

\section{GSH no Fígado pós-HP (T2)}

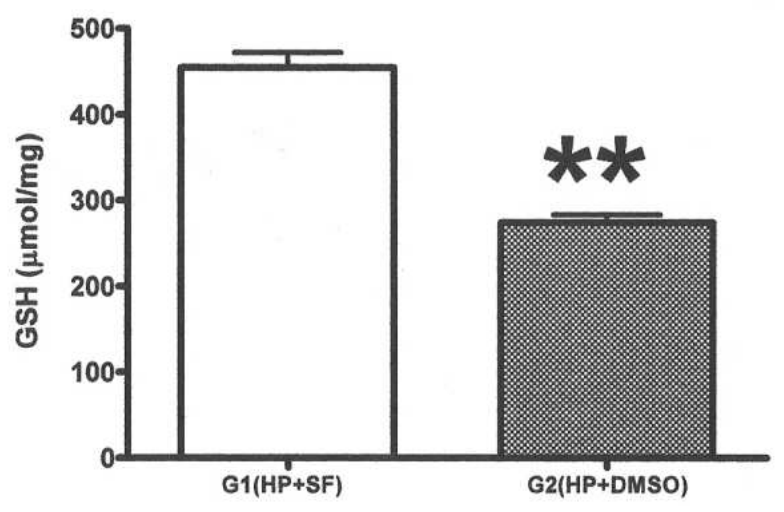

Figura 2 - A administração de DMSO resultou em queda significante $(p<0,01)$ de GSH hepático o tempo T2 pós-HP.

\section{DISCUSSÃO}

O rato foi o animal de experimentação escolhido por ser o mamífero mais estudado no fenômeno da regeneração hepática ${ }^{23}$, ter baixo custo tanto de aquisição como de manutenção e apresentar elevada resistência à infecção e ao trauma cirúrgico $^{24}$. Optou-se por animais jovens visto que a idade pode comprometer a resposta regenerativa pós-hepatectomia parcial $^{25} \mathrm{e}$ a escolha foi por sexo masculino visando atenuar a reconhecida influência dos estrógenos na regeneração hepática $^{26}$. O período de quatorze dias entre a hepatectomia parcial e o sacrifício dos últimos animais (tempo T3) foi estabelecido com base nas evidências experimentais que a regeneração hepática pós-HP nos ratos se completa em, no máximo, duas semanas, podendo inclusive ocorrer muito antes ${ }^{2,23}$. A decisão de liberar a alimentação e a água no pré-operatório repousou nos trabalhos que sustentam que o jejum anterior aos procedimentos cirúrgicos aumentam o estresse oxidativo em ratos Wistar adultos ${ }^{27}$. Apesar da anestesia inalatória com vapores de éter dietílico, método utilizado nas hepatectomias realizadas, resultar em aumento da peroxidação lipídica - de forma independente e aditiva ao fator jejum ${ }^{27}$ - e consequientemente, ser ela própria fator incentivador de estresse oxidativo, tal variável foi minimizada pelo pequeno período do ato cirúr-

\section{GSH no Fígado pós-HP (T3)}

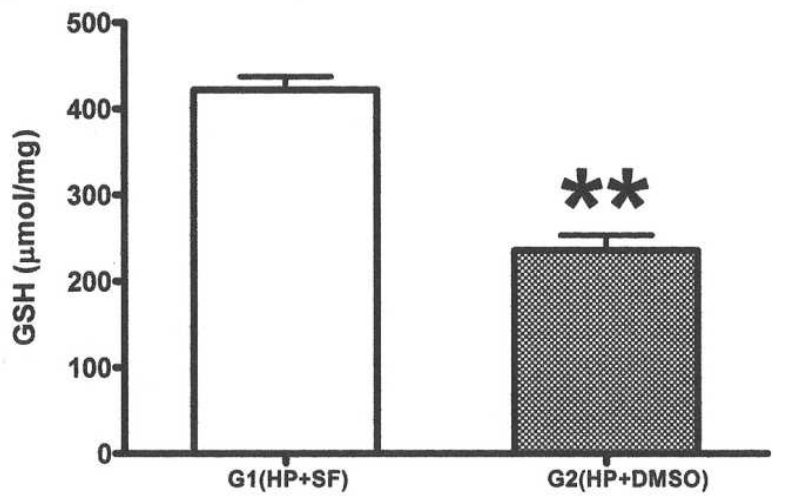

Figura 3 - Houve diminuição significante $(p<0,01)$ no GSH hepático no tempo T3 com a infusão de DMSO.

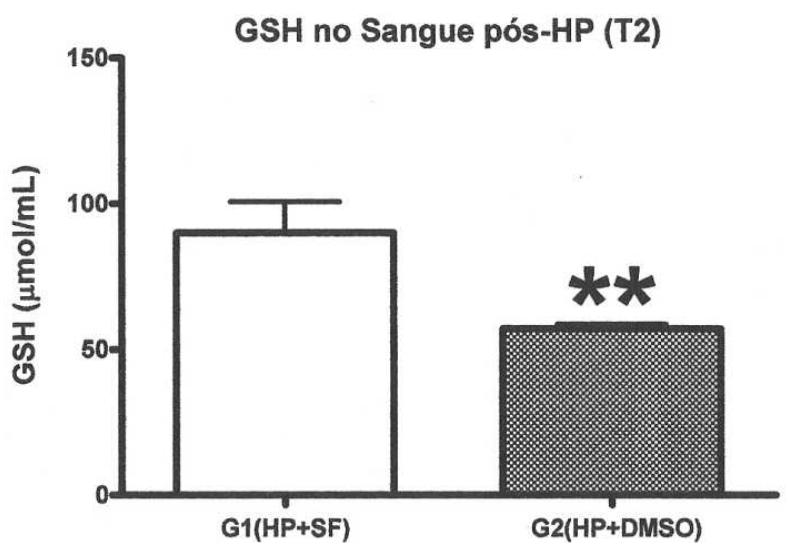

Figura 4-GSH sangüineo apresentou queda significante ( $p<0,01)$ no tempo T2 com a oferta de DMSO i.p. 


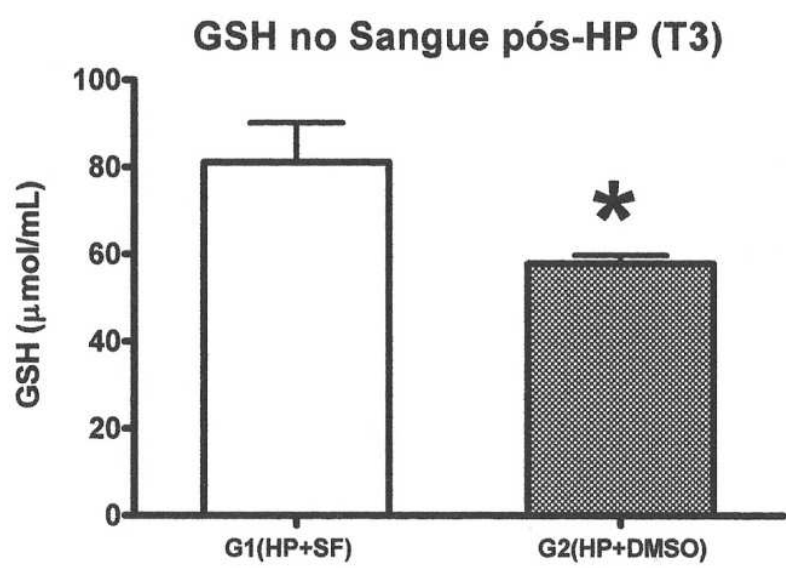

Figura 5 - GSH no sangue apresentou concentração diminuída de modo significante $(p<0,05)$ em T3 com a infusão de DMSO.

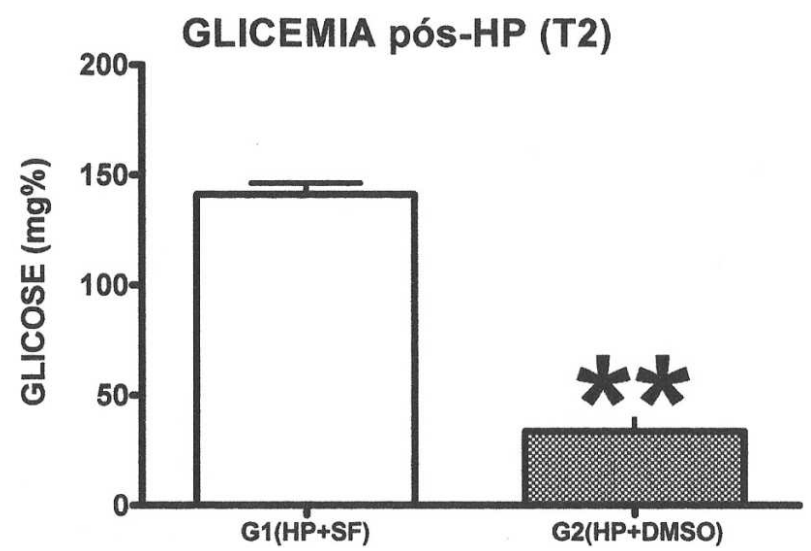

Figura 6-Significante $(p<0,01)$ hipoglicemia ocorreu nos animais sob infusão de DMSO no tempo T2.

gico (5 minutos, em média) e ser constituinte universal nos dois grupos, experimental e controle (G2 e G1, respectivamente). A via intraperitoneal foi a escolhida porque esta é uma rota clássica de administração de DMSO quando utilizado como diluente de outras drogas ${ }^{28}$.

O DMSO, um subproduto da indústria da madeira, tem se apresentado ao longo das últimas cinco ou seis décadas, entre outras propriedades, como um antiinflamatório ${ }^{29,30}$, analgésico tópico ${ }^{31}$, atenuante dos efeitos do extravasamento de quimioterápicos antineoplásicos ${ }^{32,33}$, protetor hepático das lesões causadas por tetracloreto de carbono ${ }^{34}$, protetor renal da injúria por sais de mercúrio ${ }^{35}$, inibidor de metástases ${ }^{36}$ e, principalmente, antioxidante ${ }^{17,18}$. Mas não há um consenso quanto ao volume, diluição e quantidade total de DMSO utilizados em animais experimentais. A escolha por DMSO a 3\% foi pela freqüência com que esta diluição é usada nas pesquisas.

Os antioxidantes, de um modo geral, inibem a regeneração hepática ${ }^{8,37}$. A oferta de GSH exógena, por exemplo, influi negativamente sobre a regeneração hepática ${ }^{38}$.

O DMSO aqui confirmou seu caráter antioxidativo vez que o GSH hepático apresentou queda estatisticamente significante no grupo experimento nos tempos T2 e T3, e fenômeno completamente similar ocorreu com o GSH plasmático nos mesmos tempos. $\mathrm{O}$ fato de não ter ocorrido também no tempo T1, 36h após a HP, se deve provavelmente aos aportes de DMSO, os quais só tinham ocorrido duas vezes (doses diárias) e suas presenças ainda não se faziam sentir. Mas nas mensurações de T2 e T3, as dosagens diárias de DMSO fizeram GSH "menos necessário". Via de regra, as células reagem ao estresse oxidativo com um aumento em seu conteúdo de glutationa como parte de sua resposta adaptativa à potencial lesão oxidativa.

Mesmo sob regeneração, os estudos são basicamente unânimes em mostrar que a maioria, senão todas, das funções hepáticas necessárias à homeostase orgânica, incluindo síntese de albumina, de fatores de coagulação e de bile, manutenção da glicemia e da atividade do ciclo da uréia, permanecem preservadas ${ }^{23,27}$. De fato, neste experimento, a bilirrubinemia total não apresentou qualquer distúrbio estatisticamente significante. No que se refere à glicemia, no entanto, significante redução ocorreu no grupo teste nos tempos T2 e T3 (a Figura 6 mostra o fato em T2). Tal diminuição reflete provavelmente a atuação antioxidativa do DMSO, visto que os antioxidantes possuem a capacidade de melhorar a ação da insulina, o que é explicado, em parte, pela proteção das células b produtoras de insulina das agressões dos radicais livres ${ }^{39,40,41}$.

Neste trabalho, portanto, DMSO diminuiu o estresse oxidativo em ratos sob regeneração hepática induzida por hepatectomia parcial mas não interferiu de modo significante no fenômeno da regeneração.

\begin{abstract}
Background: The purposes of this study were to evaluate dimethylsulfoxide (DMSO) influences on oxidative stress and rat liver regeneration after partial hepatectomy $(\mathrm{PH})$. Methods: 36 young male Wistar rats were randomly assigned to two groups of 18 animals each - experimental (G2) and control (G1) - and all of them were submitted to PH at the time TO. The G1 rats received daily for 14 days, by intraperitoneal (i.p.) route, $\mathrm{NaCl} 0.9 \%$ (saline) $0.1 \mathrm{~mL} / \mathrm{kg}$ and the G2 animals got daily DMSO $3 \% 0.1 \mathrm{~mL}$ $\mathrm{kg}$ by the same route. In each group, at 36h(T1), 168h(T2) and 336h(T3) post-PH, a subgroup of 6 rats were chosen in a randomized way to complementary hepatectomy, when blood and the residual liver lobes were taken to measure reduced glutathione (GSH) (blood plasma and liver) and blood concentrations of glucose and total bilirubin. All surgical procedures were performed under inhaled ether anesthesia. Results: DMSO inhibited the liver and the plasma GSH concentrations after 7 days but did not show any significant effect on liver regeneration phenomenona. Conclusion: DMSO administration leads to an inhibitory effect on oxidative stress but did not show any change on the liver regeneration evolution in rats after PH.
\end{abstract}

Key words: Hepatectomy; Oxidative stress; Rats; Liver regeneration; Lipid peroxidation; Dimethyl sulfoxide. 


\section{REFERÊNCIAS}

1. Kountouras J, Boura P, Lygidakis NJ. Liver regeneration after hepatectomy. Hepatogastroenterology. 2001; 48(38):556-62.

2. Higgins GM, Anderson RM. Experimental pathology of the liver. I. Restoration of the liver of the white rat following partial surgical removal. Arch Pathol. 1931; 12:186-202.

3. Hockings PD, Roberts T, Campbell SP, Reid DG, Greenhill RW, Polley SR, Nelson P, Bertram TA, Kramer K. Longitudinal magnetic resonance imaging quantitation of rat liver regeneration after partial hepatectomy. Toxicol Pathol. 2002; 30(5):606-10.

4. Minuk GY. Hepatic regeneration: if ain't broke, don't fix it. Can J Gastroenterol. 2003; 17(7):418-24.

5. Biondo-Simões MLP, Pante ML, Garcia RF, Macedo VL, Moraes THC, Boell P. Hormônio de crescimento na regeneração hepática em ratos. Rev Col Bras Cir. 2000; 27(2):117-20.

6. Pahlavan PS, Feldmann RE Jr., Zavos C, Kountouras J. Prometheus' challenge: molecular, cellular and systemic aspects of liver regeneration. J Surg Res. 2006; 134(2):238-51. Epub $2006 \mathrm{Feb} 3$.

7. Ronco MT, deAlvarez ML, Monti J, Carrillo MC, Pisani G, Lugano MC, Carnovale CE. Modulation of balance between apoptosis and proliferation by lipid peroxidation (LPO) during rat liver regeneration. Mol Med. 2002; 8(12):808-17.

8. Trejo-Solís C, Sánchez VC, Aranda-Fraustro A, Sánchez-Sevilla L, Gómez-Ruíz C, Hernández-Muñoz R. Inhibitory effect of vitamin $\mathrm{E}$ administration on the progression of liver regeneration induced by partial hepatectomy in rats. Lab Invest. 2003; 83(11):1669-79.

9. Ok E, Yilmaz Z, Karakücük I, Akgün H, Sahin H. Use of live oil based emulsions as an alternative to soybean oil based emulsions in total parenteral nutrition and their effects on liver regeneration following hepatic resection in rats. Ann Nutr Metab. 2003; 47(5):221-7.

10. Bannister JV, Bannister WH, Rotils G. Aspects of the structure, function and applications of superoxide dismutase. CRC Crit Rev Biochem. 1987; 22(2):111-80.

11. Jacob SW, Herschler R. Introductory remarks: dimethyl sulfoxide after twenty years. Ann N Y Acad Sci. 1983; 401: XIII-XVII.

12. Santos NC, Coelho JF, Silva JM, Saldanha C. Multidisciplinary utilization of dimethyl sulfoxide: pharmacological, cellular, and molecular aspects. Biochem Pharmacol. 2003; 65(7):1035-41.

13. Rammler DH, Zaffaroni A. Biological implications of DMSO based on a review of its chemical properties. Ann N Y Acad Sci. 1967; 141(1):13-23.

14. Kolb KH, Jaenicke G, Kramer M, Schulze PE. Absorption, distribution, and elimination of labeled dimethyl sulfoxide in man and animals. Ann N Y Acad Sci. 1967; 141(1):85-95.

15. Szmant HH. Physical properties of dimethyl sulfoxide and its function in biological systems. Ann NY Acad Sci. 1975; 243(1):203.

16. Matsumoto J. Clinical trials of dimethyl sulfoxide in rheumatoid arthritis patients in Japan. Ann N Y Acad Sci. 1967; 141(1):5608.

17. Panganamala RV, Sharma HM, Heikkila RE, Geer JC, Cornwell DG. Role of hydroxyl radical scavengers dimethyl sulfoxide, alcohols and methional in the inhibition of prostaglandin biosynthesis. Prostaglandins. 1976; 11(4):599-607.

18. Bruck R, Shirin H, Aeed H, Matas Z, Hochman A, Pines M, Avni Y. Prevention of hepatic cirrhosis in rats by hydroxyl radical scavengers. J Hepatol. 2001; 35(4):457-64.

19. Mathew T, Karunanithy R, Yee MH, Natarajan PN. Hepatotoxicity of dimethylformamide and dimethylsulfoxide at and above the levels used in some aflatoxin studies. Lab Invest. 1980; 42(2):257-62.
20. Sedlak J, Lindsay RH. Estimation of total protein-bound, nonprotein sulphydryl group in tissue with Ellman's reagent. Anal Biochem. 1968; 25(1):192-205.

21. Meites S. Bilirubin, direct reacting and total, modified MalloyEvelyn method. In: Faulkner WR, Meites S, editors. Selected Methods of Clinical Chemistry. Washington, DC: American Association of Clinical Chemistry; 1982. p.119-24.

22. Slein apud Vasconcelos PRL. Hepatic metabolism during sepsis [dissertation]. Oxford: Oxford University; 1987.

23. Ramalho FS, Ramalho LNZ, Zucoloto S, Castro e Silva O Jr. Regeneração hepática. In: Castro e Silva O Jr., Zucoloto $S$, Beer A Jr., editores. Modelos experimentais de pesquisa em cirurgia. São Paulo: Robe Editorial; 1998. p. 243-58.

24. Festing MFW. Suitability of the rat for different investigations. In: Altman PL, Katz DD, editors. Inbred and genetically defined strains of laboratory animals. Part 1: mouse and rat. Bethesda: Fed Am Soc Exper Biol. 1979; 1:237-8.

25. Bucher NLR, Swaffield MN, Ditroia JF. Influence of age upon the incorporation of thymidine-2- $\mathrm{C}^{14}$ into the DNA of regenerating rat liver. Cancer Res. 1964; 24:509-12.

26. Francavilla A, Polimeno L, DiLeo A, Barone M, Ove P, Coetzee M, Eagon P, Makowka L, Ambrosino G, Mazzaferro V, Sarzl TE. The effect of estrogen and tamoxifen on hepatocyte proliferation in vivo and in vitro. Hepatology. 1989; 9(4):61420.

27. Liu PT, Kentish PA, Symons AM, Parke DV. The effects of ether anaesthesia on oxidative stress in rats - dose response. Toxicology. 1993; 80(1):37-49.

28. Rao VS, Santos FA, Sobreira TT, Souza MF, Melo CL, Silveira ER. Investigations on the gastroprotective and antidiarrhoeal properties of ternatin, a tetramethoxyflavone from Egletes viscosa. Planta Med. 1997; 63(2):146-9.

29. Shirley SW, Stewart BH, Mirelman S. Dimethyl sulfoxide in treatment of inflammatory genitourinary disorders. Urology. 1978; 11(3):215-20.

30. Santos L, Tipping PG. Attenuation of adjuvant arthritis in rats by treatment with oxygen radical scavengers. Immunol Cell Biol. 1994; 72(5):406-14.

31. Evans MS, Reid KH, Sharp JB Jr. Dimethylsulfoxide (DMSO) blocks conduction in peripheral nerve $\mathrm{C}$ fibers: a possible mechanism of analgesia. Neurosci Lett. 1993; 150(2):145-8.

32. Alberts DS, Dorr RT. Case report: topical DMSO for mitomycin C-induced skin ulceration. Oncol Nurs Forum. 1991; 18(4):693-5.

33. Lebredo L, Barrie R, Woltering EA. DMSO protects against adriamycin-induced tissue necrosis. J Surg Res. 1992; 53(1):625.

34. Wong C, Ooi V, Wong C. Effects of dimethyl sulphoxide against liver injury caused by carbon tetrachloride in rats. Toxicol Mech Methods. 2004; 14(3):167-76.

35. Jo SK, Hu X, Yuen PS, Aslamkhan AG, Pritchard JB, Dear JW, Star RA. Delayed DMSO administration protects the kidney from mercuric chloride-induced injury. J Am Soc Nephrol. 2004; 15(1):2648-54.

36. Salim AS. Removing oxygen-derived free radicals delays hepatic metastases and prolongs survival in colonic cancer. A study in the rat. Oncology. 1992; 49(1): 58-62.

37. Kirimlioglu V, Kirimlioglu H, Yilmaz S, Ozgor D, Coban S, Karadag N, Yologlu S. Effect of fish oil, olive oil, and vitamin E on liver pathology, cell proliferation, and antioxidant defense system in rats subjected to partial hepatectomy. Transplant Proc. 2006; 38(2):564-7.

38. Holecek M, Skopec F, Sprongl L. Influence of buthionine sulfoximine, S-adenosylmethionine and glutathione on liver regeneration following partial hepatectomy. Arzneimittelforschung. 2000; 50(12):1093-8. 
39. Paolisso G, D'Amore A, Giugliano D, Ceriello A, Varricchio M, D'Onofrio F. Pharmacologic doses of vitamin E improve insulin action in healthy subjects and non-insulin dependent diabetic patients. Am J Clin Nutr. 1993; 57(5):650-6.

40. Barbagallo M, Dominguez LJ, Tagliamonte MR, Resnick LM, Paolisso G. Effects of vitamin E and glutathione on glucose metabolism. Hypertension. 1999; 34(4 Pt 2):1002-6

41. Facchini FS, Humphreys MH, Nascimento CA, Abbasi F, Reaven GM. Relation between insulin resistance and plasma concentrations of lipid hydroperoxides, carotenoids, and tocopherols. Am J Clin Nutr. 2000; 72(3):776-9.
Como citar este artigo:

Melo JU, Vasconcelos PR, Santos JM, Campos MM Jr, Barreto MV, Kimura OS. Efeitos do dimetilsulfóxido no estresse oxidativo e na regeneração hepática pós-hepatectomia em ratos. Rev Col Bras Cir. [periódico na Internet] 2008; 35(2). Disponível em URL: http:/ /www.scielo.br/rcbc

Endereço para correspondência:

José Ulisses de Souza Melo

Rua Afonso Celso, 897 - Aldeota

Fortaleza - Ceará

CEP: 60140-190 